\title{
AC 2012-4956: CLOUD COMPUTING IN COMPUTER SCIENCE AND ENGINEERING EDUCATION
}

Prof. Hassan Rajaei, Bowling Green State University

Hassan Rajaei is a professor of computer science at Bowling Green State University, Ohio. His research interests include cloud computing, high performance computing, distributed simulation, parallel and distributed processing, communication networks, wireless communications, and virtual training environments. Rajaei received his Ph.D. from Royal Institute of Technology, KTH, Stockholm, Sweden, and he holds a M.S.E.E. from the University of Utah.

Eman A. Aldakheel, Bowling Green State University 


\title{
Cloud Computing in Computer Science and Engineering Education
}

\begin{abstract}
Cloud Computing opens a new chapter in Information Technology. It has its roots in internet technology, and like the Internet, it is rapidly and forcefully advancing into a large range of applications and services. While standardization of Cloud Computing is ongoing, there is every indication that cloud technology is here to stay and will cover most sectors of the society, including education. This paper discusses the potentials of Cloud-Based Education (CBE) in STEM areas to better stimulate and engage students in their pursuit of knowledge and learning.

This paper introduces the concept of Cloud-Based Education for Computer Science (CBE-CS) and discusses how its framework can be achieved. Further, it shows how the platform can be generalized to use in various STEM areas. The authors argue that the potentials in using Cloud Computing for teaching Computer Science courses are extraordinary since CS has an intimate relationship with the cloud infrastructure. Thus, CBE-CS can greatly facilitate teaching complex underlying organizations of CS courses such as Operating Systems, Communication Networks, Cyberspace Security, Web-Based Applications, Database, and High Performance Computing. While other STEM education can extensively benefit from CBE at the Software as a Service (SaaS) level to present meaningful examples in the lectures, CS courses can move deeper and utilize also the lower levels of cloud services. Such capability can deliver valuable examples and laboratories for CS students to better understand large scale applications and their complexities.
\end{abstract}

\section{Introduction}

The accelerated development in Information Technology and the necessity for enhanced learning environments by harnessing advance technologies and resources has created a need to teach Computer Science and Engineering students more effectively using technological advances. Cloud Computing ${ }^{1,2,3,4,5,7}$ appears to be one of the most beneficial tools for this purpose due to the offered benefits like availability, scalability, agility, elasticity, and on-demand services. These characteristics, among many others, can give the impression that Cloud Computing might significantly enhance the learning environment especially for STEM.

Cloud Computing evolved from virtualization technologies already existed in the IT ecosystem. Virtualization hides the underlying complexities and allows multiple customers, with diverse applications and demands, to utilize the Cloud simultaneously. The platform can offer a wide range of services using the virtualized services. The Cloud delivers a unique view of its resources and services, promoting user friendliness, cost-effectiveness, elasticity, and the pay-as-you-go business model. These features triggered significant interests in Cloud Computing worldwide.

One important service of the Cloud can be regarded as Cloud-Based Education. This capability can promote cloud services in educational settings with minimal interference from the local data centers. Consequently, the cost of the needed infrastructure is reduced and the availability of 
establishing an advanced educational environment is dramatically augmented. For this reason and many others, Cloud Computing is poised to become a game changer, especially in education. With access to the Cloud, an educational institution can quickly develop and deliver advanced distance learning, with excellent benefits to STEM education. Quick availability of complex computations and visualization of applications in diverse STEM domains such as chemistry, biology, physics, mechanics, and others can become a reality for the educators and the learners.

The ecosystem of a typical cloud utilizes a three-layered architecture ${ }^{2,7}$. On the top layer, there is Software as a Service (SaaS). In the second layer, there is Platform as a Service (PaaS) and in the third layer, Infrastructure as a Service (IaaS). A CBE-CS platform will utilize all three layers offering new services to the educational community. This paper examines how specific Computer Science courses can be mapped to certain layer(s) of the Cloud (see Sections 4 \& 5).

Rapid growth of the cloud technology and promises to reduce the IT costs ${ }^{5}$ pushed numerous educational institutions to revise their IT infrastructure and follow the Cloud development. Our university, like many others, is moving to the Cloud within the next five to ten years. Computer Sciences and STEM Education are among prime targets.

Ever since the emergence of Cloud Computing, significant emphasis and efforts have been offered in exploring and defining this new technology ${ }^{4,7,8,9}$. Experts predict that by 2020 most institutions and enterprises will move to the Cloud eliminating the dependency of desktops ${ }^{10}$. Nevertheless, the universal scheme among all Clouds is the scalability of offered services through the Internet. These services vary, starting from the needed hardware to the applications. Several research efforts ${ }^{5}$ in educational field focused on using public clouds like Windows Azure $^{11}$, Amazon Elastic Compute Cloud (EC2) ${ }^{12}$, and Google AppEngine ${ }^{13}$. While standardization efforts are underway by NIST and others, majority of the cloud users seek the power of the Cloud in the infrastructure, platforms, software, and services.

This paper introduces and describes CBE-CS. Section 2 reviews related work. Section 3 provides an overview of Cloud Computing and CBE while CBE-CS is described in Section 4. Selected Pilot CS courses for CBE are presented in Section 5 and an implementation framework is given in Section 6. Finally, Section 7 outlines some discussions, future work, and concluding remarks.

\section{Related Work}

Cloud-Based applications for education attracted many researchers and educators. While majority of developers are targeting to move their distance learning platforms to the Cloud, few are using cloud capabilities to explore better use of the technology and its services into their lectures. Our efforts are among the latter. Our investigation indicates that most CS educators focus on certain courses and put their efforts in teaching the selected courses on the Cloud. In contrast, our focus is to integrate the Cloud capabilities into the CBE-CS platform. Unlike other Cloud-Based Education models, the proposed CBE-CS framework takes advantage of the intimate relationship that exists between the cloud technologies and CS courses. Due to this unique attribute, a CBE-CS platform can penetrate into all layers of the Cloud and provide meaningful examples for students. This section briefly reviews the work on virtual classrooms and e-learning and then examines examples of the efforts on Cloud-Based Education. 


\subsection{Virtual Classrooms}

A virtual classroom (VCR) is an interactive environment where the learners and educators collaborate via computers ${ }^{14}$. The learning setting is often augmented by multimedia to enable interactivity. With the growth of Web 2.0, social interactions have played an important role in virtual environments beyond e.g. a simple game-based learning. Unlike a traditional classroom, the content of a VCR course can be repeatedly used ${ }^{15}$. In addition, VCRs often offer two distinct features where traditional e-learning ${ }^{16}$ does not support: virtual presence, and synchronous live distribution. CBE can eliminate limitations of traditional e-learning.

\subsection{CBE Examples}

This section describes examples of efforts from others using Clouds for their learning setting. Ma et al. ${ }^{17}$ discussed suitable cloud applications to construct a collaborative learning setting. They illustrated benefits of Cloud Computing in e.g. solving storage limitation when dealing with massive resources. Further, they focused on constructing a platform to exploit the needed tools within the Cloud. To achieve this goal, four basic principles were used: learner centric, rationality of the navigation system, collaborative \& sharing of e-learning, and effectiveness of the teaching resources. They used Google Cloud for their collaborative learning platform.

EduCloud $^{18}$ (by Vaquero) focuses on the use of IaaS and PaaS in teaching advanced CS courses. In a case study, Vaquero selected eighty four students and four educators for advanced network courses to evaluate teaching on the Cloud. The study showed that lack of identifying appropriate service level of cloud abstraction can lead to problems. Nevertheless, the study showed that cloud utilization enhances students' learning outcomes. The study concluded that using the Cloud helps students to focus on their tasks and not dealing with unrelated issues. Further, the study showed that PaaS can be prevalent choice for higher level courses alleviating students not to deal with the hardware. Nonetheless, IaaS would be appropriate for course such as Operating System and Software Engineering which may need details of operations using the Virtual Machine.

Another Cloud-Based solution for e-learning is presented by Al-Zoube ${ }^{19}$. The system is a Virtual Learning Environment (VLE) and is highly interactive using different tools and technologies. The VLE content was developed by teachers. While the research has been content-centric, the environment is learner-centric and follows the learner pace.

A Virtual Computing Laboratory (VCL) was constructed by North Carolina State University using IBM Cloud. This VCL offers the students the ability to allocate and access a VM as a basic machine or a specific application image such as $\mathrm{Matlab}^{20}$.

\section{Cloud-Based Education}

This section describes what a Cloud-Based Education is and how it can utilize cloud services. To better illustrate the concept, fundamentals of Cloud Computing is presented first, then e-learning and Virtual Classrooms are explored. After the common features of a CBE have been established here, Section 4 presents CBE-CS in details and how it can be mapped into the Cloud. 


\subsection{Cloud Fundamentals}

NIST and other organizations define Cloud Computing as Internet-based technology $y^{2,6,8}$ which offers computational resources via a computer network and delivers flexible, scalable, and ondemand services to the end-users. It furnishes large storage capacity, network bandwidth, and vast processing powers ${ }^{4,6}$. The technology aims at freeing the users from being attached to specific machines. Cloud Service Providers (CSPs) assure performance of applications through Service Level Agreement ${ }^{21}$ (SLA). The services are classified into three basic layersapplications, platforms, and infrastructures —each serving different purposes and tasks ${ }^{11}$.

The first layer is referred to Infrastructure as a Service (IaaS). This layer can accommodate a complete IT infrastructure and deliver Virtual Data Center as a Service (VaaS). At this level, CSPs often offer different services, for example, computing power represented by Virtual Machines (VMs), storages, virtual servers, networking services and more. The users can obtain the needed resources delivered to them via CSPs' portals. For example, Amazon Elastic Compute Cloud (Amazon EC2) ${ }^{12}$ provides computing capacity via Amazon's data centers. IaaS offers a scalable infrastructure with the rapid provisioning feature. In addition, the users pay only for the utilized resources for the time period they needed eliminating overspending capital and initial setups. By integrating different clouds to offer additional resources, institutions may no longer be worried about resource limitations. This characteristic can play a key role in CBE in general and CBE-CS in particular as laboratories can be setup and torn down when they are needed.

The second layer is referred to Platform as a Service (PaaS), which provides the ability to develop and deploy applications. CSPs often offer set of tools and services to help the developers to build their applications. Further, they can use plug-ins or prebuilt sub-programs for any Webbased applications which are compatible with the CSP's applications. PaaS offers services in different abstractions. The clients can use existing sets of deployed applications considered as high-level services, or build platforms using the provided operating systems and middleware.

The third layer is on the top of PaaS and is referred to Software as a Service (SaaS). It aimed to deliver easy access to cloud applications through the Internet. In this layer, a CSP can provide a single instance on the Cloud for multiple users. Google Apps, a popular SaaS domain ${ }^{13}$, provides variety of Web-Based applications for business, education, and government.

Figure 1 shows examples of cloud services with selected CSPs in respect to the service layers. IaaS can deliver servers, storage, connectivity, load balancing, and firewall services. As shown, GoGrid ${ }^{22}$, Amazon and others provide IaaS services. The PaaS layer acts as a container enabling the users to modify and develop their platform and deploy their applications. Typical CSP at this layer are Force.com, Windows Azure, and Google AppEngine. The most abstract layer of the cloud service is SaaS, which enables users to run hosted applications on the Cloud and use them remotely, for example Yahoo mail and Gmail. SalesForce ${ }^{23}$ is another example, which is a popular provider of Customer Relation Management (CRM) applications. In Section 4, we use the model presented in Figure 1 to map CBE-CS platform and illustrate how certain CS courses can be mapped to certain layers of the Cloud architecture. 


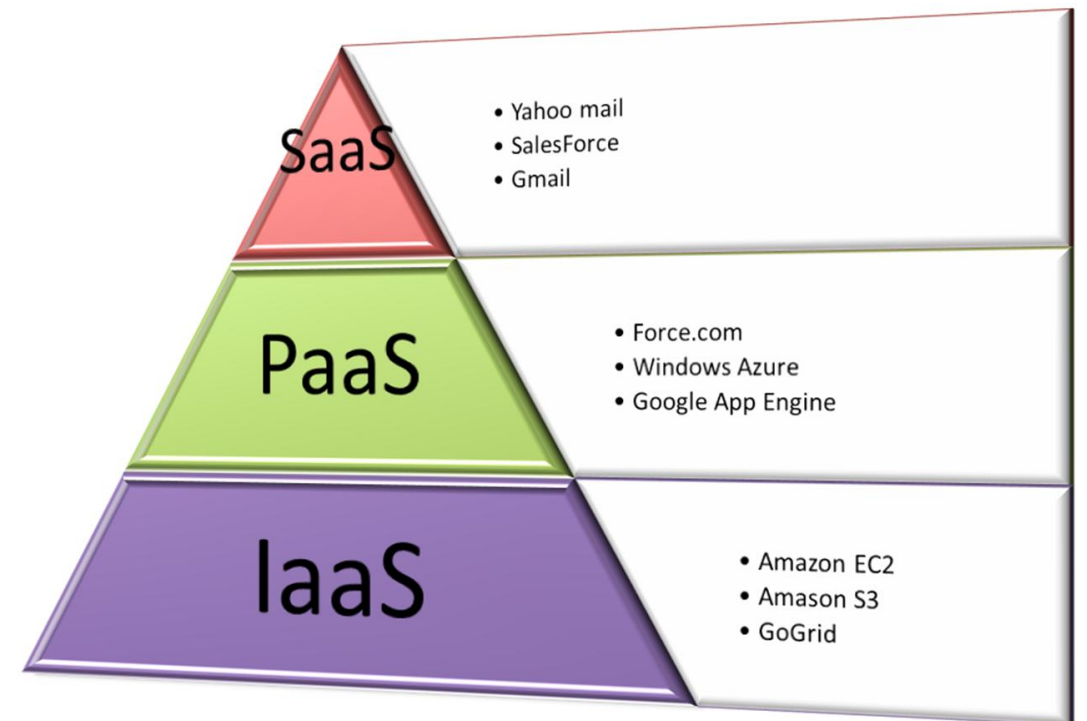

Figure 1: Basic Service Layers of Cloud Computing with selected Cloud Service Providers (CSPs) for each layer.

\subsection{E-learning and the Cloud}

Distance Learning (DL), also known as e-learning, has an important role in education, especially for students who have health, work, location, or disabilities that hinder them to attend a traditional classroom. Students can join diverse activities online to meet the learning objectives of a class ${ }^{24}$. Despite the offered flexibilities, e-learning has limitations and challenges ${ }^{24,25}$. Examples include scalability, quality, real-time interaction, and affordability due to the space restriction and the technology used. Traditional e-learning uses the client/server model often with limited network bandwidth. When excess requests sent to the server, it gets congested. Most traditional e-learning systems lack social interactions caused by restricted technology with marginal quality of service. A 2006 study on e-learning market estimated reaching to $\$ 28$ billion by $2008^{26}$. In that year, the synchronous e-learning portion was worth $\$ 5$ billion (only $18 \%$ ) whereas in 2011 it was doubled ${ }^{27}$. Cloud services have alleviated several shortcomings of elearning infrastructures. As a result, it is anticipated that Cloud-Based Distance Learning will be expanded dramatically in future due to the lower cost of maintenance, the elasticity features, and the pay-as-you-go business model.

\subsection{Cloud as a Educational Platform}

One important feature of the Cloud is elasticity ${ }^{2,4,6,12}$. This is a result of virtualization which gives the illusion of having unlimited resources available at the client leisure time.

Cloud-Based Education, also known as a blended e-learning ${ }^{27,}{ }^{28}$, serves as a complementary vehicle to a face-to-face class to enhance students learning outcomes ${ }^{28}$. In CBE, students attend the class similar to face-to-face classrooms while using advanced e-learning surrounding that delivers learning materials via multiple technologies (Figure 2). CBE builds the learning environment by utilizing cloud services. The platform has dynamic capabilities and is scalable. The learning ecosystem of an institution can be customized by using the IaaS and PaaS services. 
On top of that, SaaS can deliver the learning contents and eliminate software updating, licensing, and maintenance. Incorporating traditional face-to-face learning with tools hosted on the Cloud can improve the traditional learning settings ${ }^{29}$.

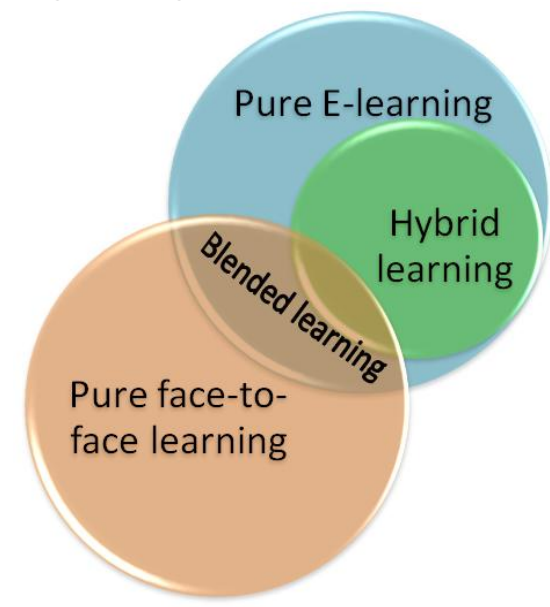

Figure 2: Illustration of diverse educational settings and blended learning

CBE has potentials to overcome several shortcomings faced in the traditional e-learning systems. It reduces the costs and time of administration, maintenance, and operations while increasing the availability of the resources for the learners. In addition, CBE offers the ability for institutes which may not afford the initial costs to build an effective e-learning ecosystem ${ }^{30}$. Using the Cloud to build institutional infrastructure for virtual classrooms is beneficial to all stakeholders. Scalability and reliability of the framework are among the top advantages of this e-learning technique. Figure 3 shows the benefits of a CBE system for three categories of users: Educator, Administrator, and Learners. For each category potential benefits are exposed in Figure 3.

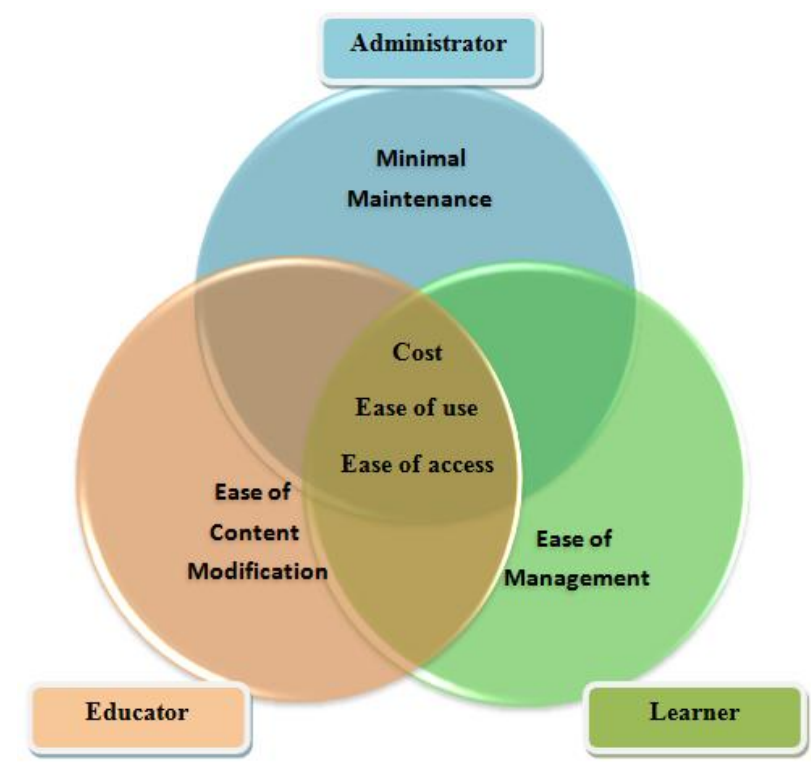

Figure 3: Cloud-Based Education benefits to the stakeholders (as viewed by the authors). 


\section{CBE for Computer Science and Engineering Education}

The goal is to deliver an improved learning setting for computer science education. Using the $\mathrm{CBE}$ concept, the framework can become portable and be generalized to offer a wider range of services for computer science and engineering education.

To determine potential computer science courses, we analyze our curriculum in respect to the CS2008 Review Taskforce ${ }^{31}$ sponsored by ACM and IEEE Computer Society. The analyzing process was based on the assignment types, syllabus components, and course specifications in the CS Curriculum $2008^{31}$, and with respect to the base services of Cloud Computing. The result is illustrated in Figure 4 mapped into the three layers of the Cloud architecture. We trust the presented mapping facilitates eloquent learning outcomes.

This work examined Cloud services to determine which courses would suit best to what level. The goal has been to teach the content in an interactive mode to better stimulate students learning. It was decided to spotlight the non-theory courses in this effort since most cloud services use programming. This is the focal point identifying the intimate relationship between Cloud Computing and Computer Science education. The selection does not outcast theory courses but rather stresses the attention to utilize PaaS services. Clearly, any CBE can benefit from SaaS which ultimately uses IaaS utilities. In addition, the project identified different levels of difficulties in courses to explore more than one layer of cloud services.

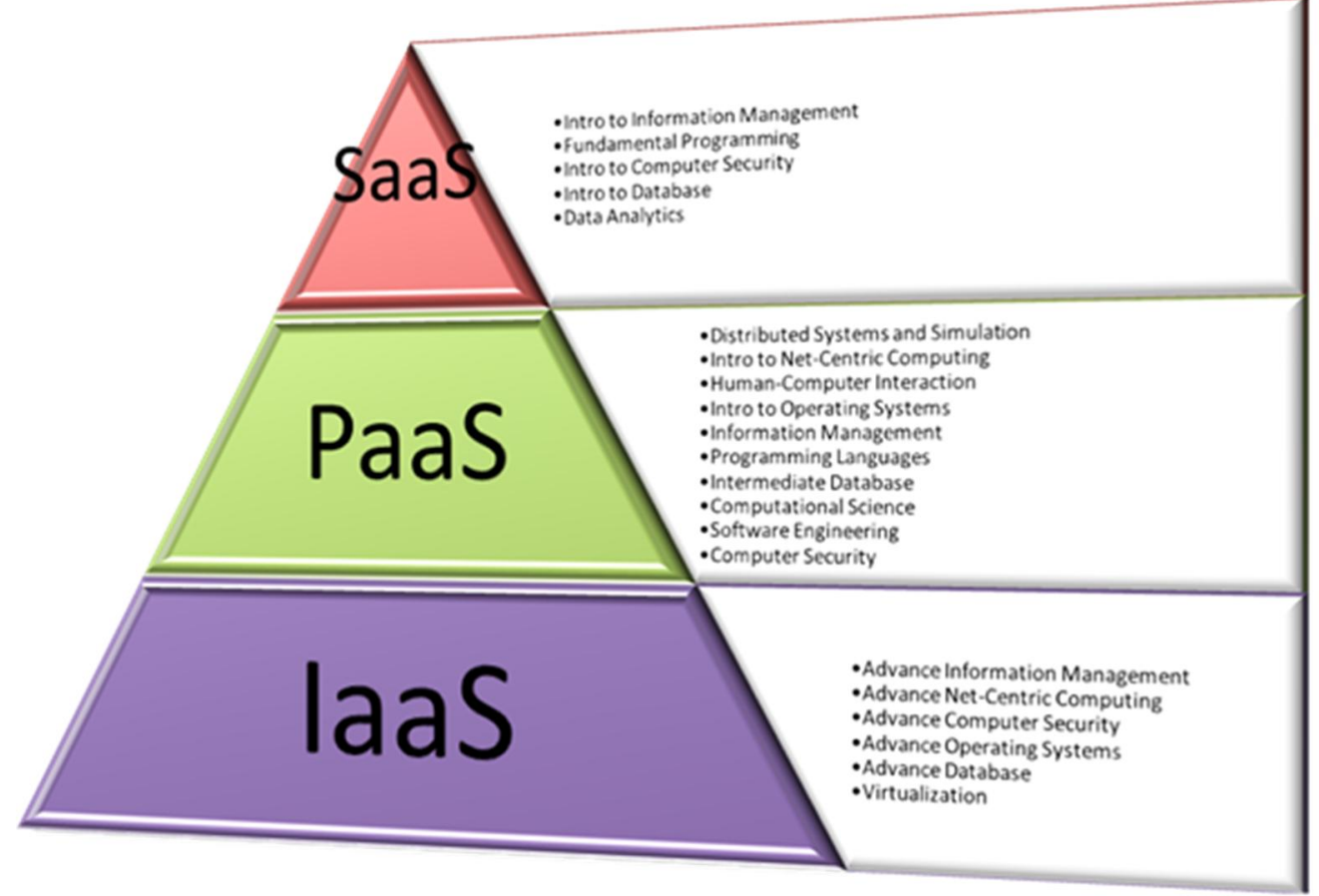

Figure 4: The proposed CBE-CS architecture mapping Computer Science courses to the Cloud architecture (courses appearing in multiple layers have different levels).

Moving from the SaaS layer to the PaaS reflects the increase in the depth, difficulty, and complexity of a course. Likewise, the move from PaaS to IaaS indicates complexity of a course with respect to the needs for details in VM configuration, networking, and operating systems. 
For course like Fundamental Programming, Intro to Computer Security, Data Analytics, and other courses on SaaS layer, we propose using hosted applications on the Cloud and eliminate licensing issues, maintenance, and updates. For Fundamental Programming courses, any programming language hosted on the Cloud can be used. Managing user access and group organization can be done at SaaS level for e.g. Intro to Computer Security course.

\section{Pilot Courses and Framework}

In this paper, three CS courses are selected to demonstrate potentials of a CBE-CS platform: Database, Operating Systems, and Parallel Programming. The descriptions below on these pilot cases can easily be expanded for other CS courses indicated in Figure 4.

\subsection{Database}

Most clouds offer database services. Students can use the services and create their databases, connect them together, implement and maintain rational databases, and retrieve information using Structured Query Language (SQL) with PHP or other Web programming language. As an example we explore IBM and Windows Azure. IBM Clouds offers a database solution by providing DB2 images. Students create their database by selecting and adding DB2 images for the desired operating system ${ }^{32}$. After naming the image, students can setup the security level by creating private and public keys configuring the DB2 image (Figure 5).

\begin{tabular}{|c|c|}
\hline 重曋䇍。 & Close $[x]$ \\
\hline \multicolumn{2}{|c|}{$\begin{array}{l}\text { Add instance } \\
\text { Step } 2 \text { of } 4: \text { configure image }\end{array}$} \\
\hline \multicolumn{2}{|c|}{$\begin{array}{l}\text { You selected: DB2 Enterprise Developer Edition } 9.7 .1 \text { 64bit } \\
\text { This image contains DB2 Enterprise component of IBM Database Enterprise Developer Edition (DEDE) } \\
\text { and is buitt on RHEL } 5.464 \text { bit. Use this image to design, prototype, develop, and test database } \\
\text { applications (non-production use). Build 3.2.sby }\end{array}$} \\
\hline \multicolumn{2}{|c|}{ Complete the fields below to configure your instance selection. Required fields are indicated with an asterisk ( $)$. } \\
\hline Request Name: & dW DB2 data server \\
\hline Quantity: & 1 \\
\hline Server Size: & Bronze 64 bit \\
\hline Expires on: & $10 / 9 / 10$ \\
\hline Key: & $\mp \oplus$ Add Key \\
\hline Select IP: & - How do ladd an IP? \\
\hline Mount Storage: & - How do l add Storage? \\
\hline Image ID: & 10010275 \\
\hline & $\rightarrow$ Next $X$ Cancel \\
\hline
\end{tabular}

Figure 5: Configuring DB2 image of IBM's Clouds ${ }^{32}$ (Krook, 2011) 
The access permissions must be identified. DB2 images give the ability to set three different access levels: owner, administrator, and user. The password should be entered for each access level. After this, students can start to write PHP and SQL codes using their username and password on the portal interface. In addition, learners can use remote access from their local machine by using the public and private keys.

Similarly, Windows Azure offers a database solution with a user friendly interface (Figure 6). To build a database with Windows Azure, students start with creating a subscription, followed by creating the server, the access levels, and passwords. The database is created via the .NET framework and connected to the server.

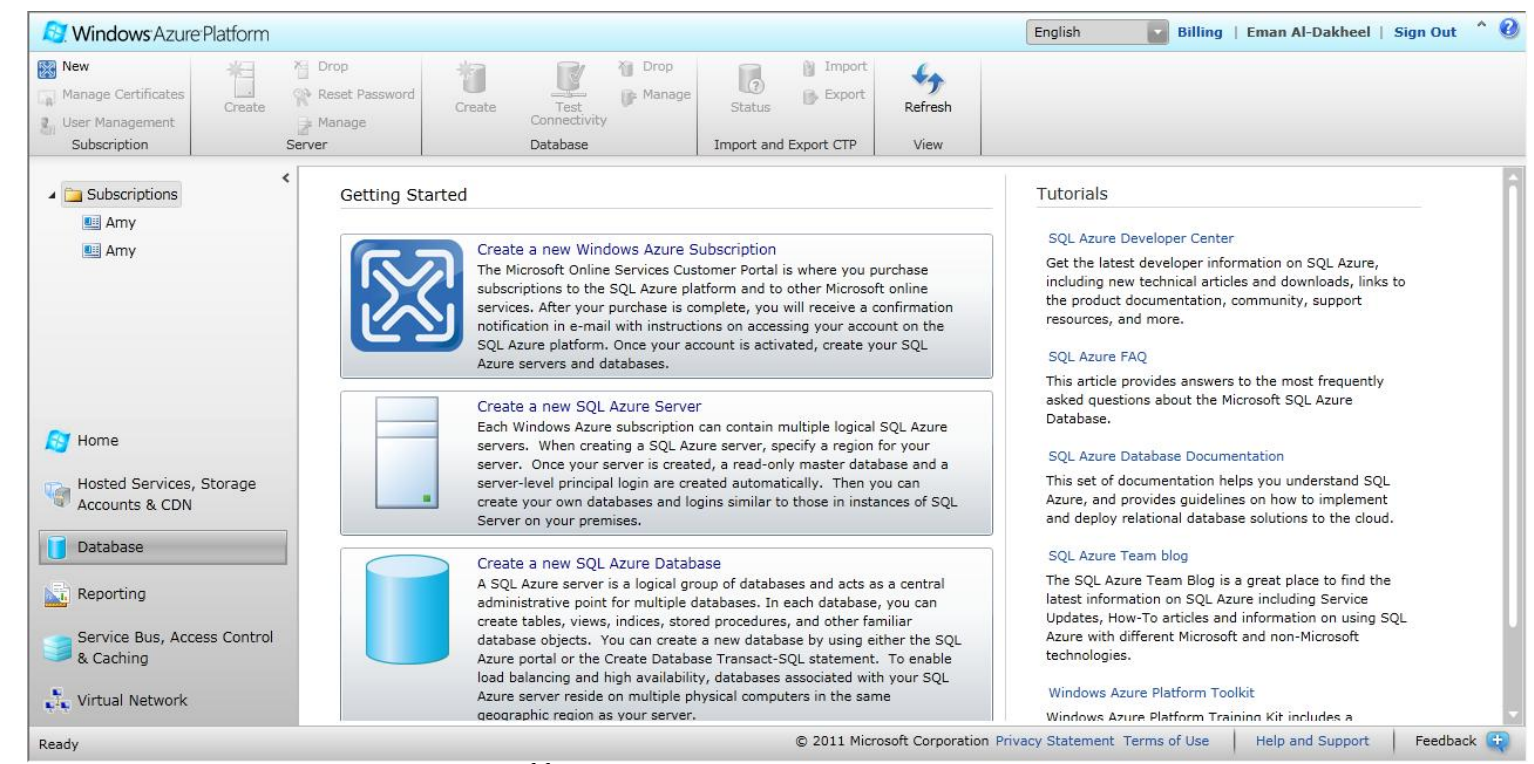

Figure 6: Windows Azure ${ }^{11}$ database solution interface snapshot (Microsoft, 2011)

\subsection{Operating Systems}

Operating Systems (OS) is one of the most beneficial courses for CBE-CS since most CSPs offer variety of OS images. Students can perform multiple exercises and programming assignments on the available OS images. In addition, they can design their own OS and implement its image in a Virtual Machine (VM) on the Cloud. Due to virtualization techniques, no harm will be done if student's version crushes. This is in contrast to a real machine which is the nightmare of any system administration to allow students having root access. VM will expose the students to the in-depth knowledge on different operating systems, their performances, and compatibility.

Students can practice the behaviors of different scheduling algorithms using any programming language, virtual memory, or device management, and so on. In a traditional setting, such exercises are only possible using simulation and not real machine. With CBE-CS, students will experience building and testing real algorithms to be executed on the Cloud.

To start programming with Amazon AWS ${ }^{12}$, the service selection comes first through the AWS interface. After the service selection, the user chooses an appropriate Amazon Machine Image (AMI) or upload a specific created image from his/her local device to AWS, as Figure 7 
illustrates. The user then needs to specify the number of instances. Finally, the wizard provides a detail report about the selected instances.

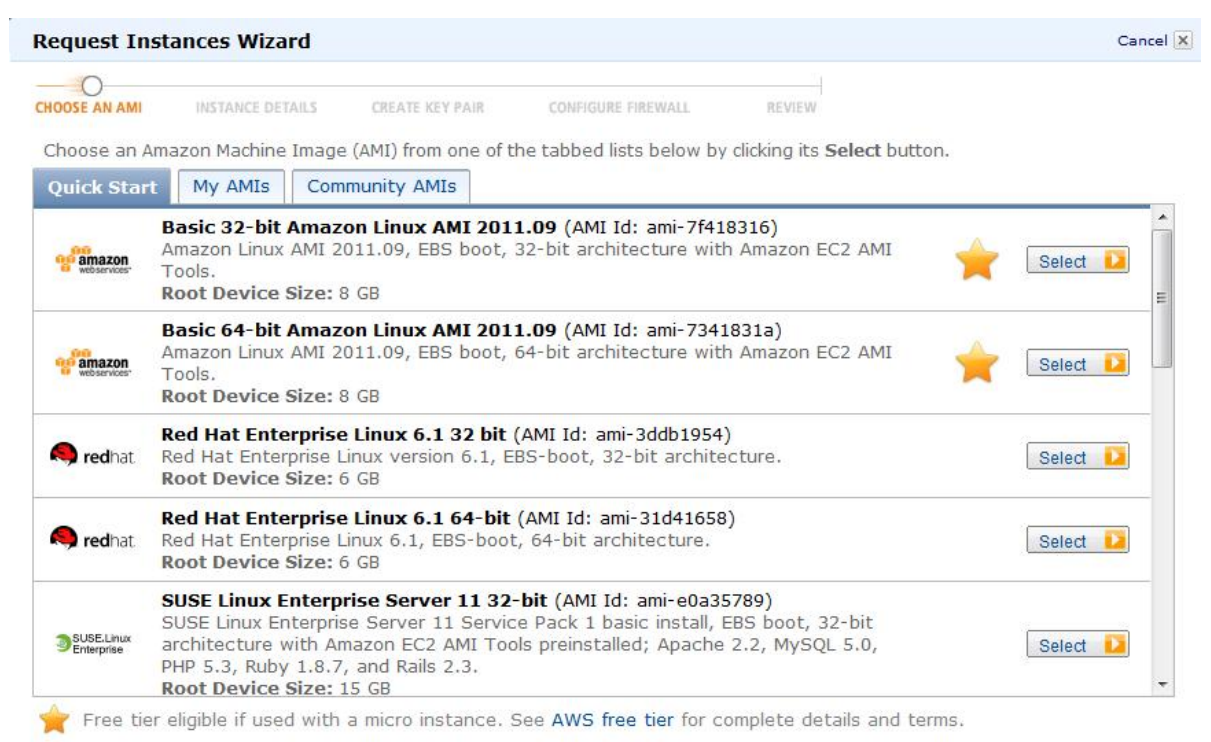

Figure 7: Available Virtual Machine images from AWS ${ }^{12}$ allowing students to work with different operating systems and implementations (Amazon, 2011).

\subsection{Parallel Programming}

One distinct advantage of CBE-CS for student learning is parallel programming which is the foundation of High Performance Computing (HPC) where the Cloud is ultimately resides. The availability of potentially unlimited processor power offers an outstanding platform for any parallel programming courses.

Cluster, Grid, and High Performance computing is one of the cornerstones of computer science curriculum. To teach students the HPC concept, one needs hands-on practices with different settings of parallel programming paradigm. With the availability of commodity cluster and multicore hardware, it is fairly easy to achieve the basic tool. Nevertheless, the initial costs, the setups, installations, and maintenance are challenging. A CBE-CS platform overcomes the availability of HPC and offers students the tool to practice their lab assignments when they need.

Students need to understand and practice with issues such as scalability, synchronization overhead, performance, partitioning, and load balancing of diverse applications. The same rule should apply whether students are using local clusters or the Cloud. However, using actual distributed servers in the cloud can improve students' understandings for issues related to the distributed system, thus improving the learning outcomes.

Numerous parallel models exist today in a parallel programming course. Examples include multi-threading (shared-memory), message-passing, distributed systems, SIMD vs. MIMD style, and recently GPU programming. Students using CBE-CS can exercise, for example, with a Message Passing Interface (MPI) and run their programs. Use of Amazon's EC2 is illustrated in 
Figure 8. Students may implement an application program using $\mathrm{MPI}^{33}$. Building MPI or OpenMP via OpenSSH ${ }^{34}$ allows students to test, run, debug, and deploy their applications on a highly scalable distributed system. This process will expose the students with e.g. hidden bugs and other issues mentioned above to understand parallel programming complexities.

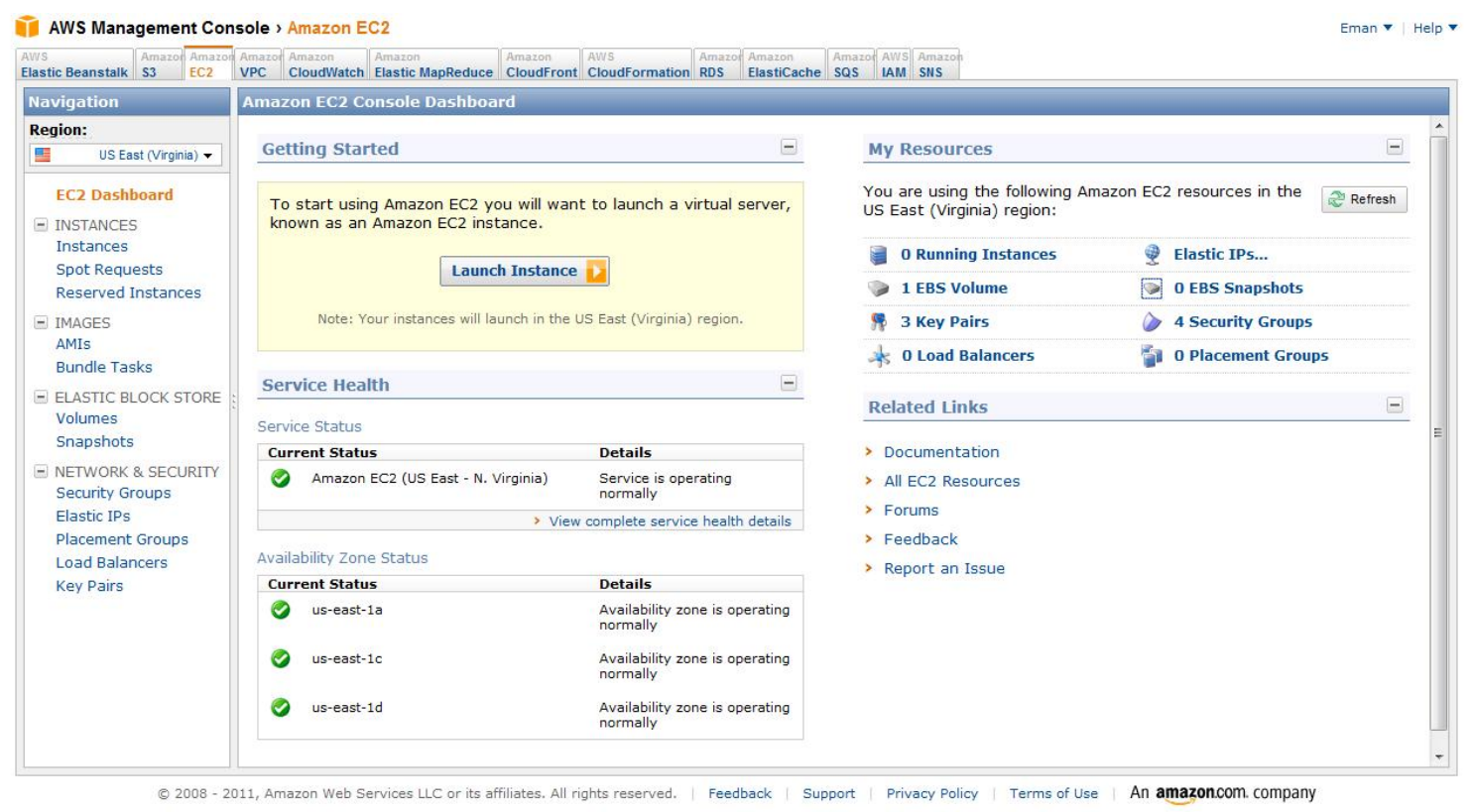

Figure 8: Amazon $\mathrm{EC} 2^{12}$ interface and services snapshot (Amazon, 2011)

\section{Implementation Framework}

As discussed earlier, Distance Learning (DL) in its current client-server mode is limited and needs to be augmented with advance features often found in advanced virtual classrooms. Harnessing the Cloud into e-learning gives more flexibilities and dynamic resource allocation. Virtual classrooms integrated with Cloud Computing helps to overcome some DL and e-leaning limitations. An abstract view of CBS-CS implementation framework is illustrated in Figure 9. In this figure, DL is at the bottom layer followed by Cloud Computing, and integrated with a virtual classroom layer. An interface will help the users to access the course contents of CBE-CS. This architecture can enhance QoS when adding more students or multimedia contents. In addition, the live video conferencing limitation, which is normally a problem in traditional systems, can be eliminated when using clouds with good bandwidth ${ }^{35}$.

Based on the identified courses and selected clouds, a CBE-CS Ecosystem has been constructed which is illustrated in Figure 10. The focus here is, how to integrate the courses on the Cloud and how the Cloud can be integrated in the teaching process. The proposed ecosystem contains four layers: User Interface, SaaS, PaaS, and IaaS. In addition, three modules are added for access, maintenance, and security: user log database, system security, and service management. This ecosystem covers the issues presented in this paper. It is fairly straightforward to generalize this system and expand it to other sciences and engineering education, especially those that need extensive computing power and visualization. Future work will target implementing the framework and carefully studying its limitations and strengths. 

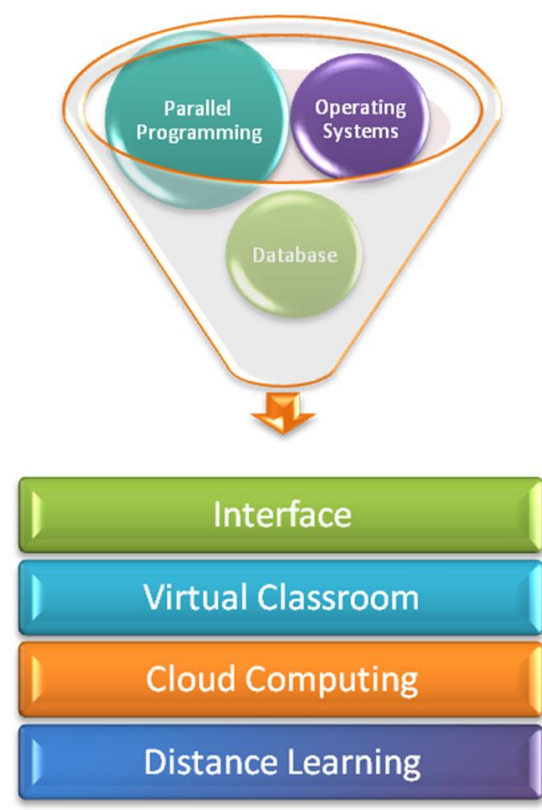

Figure 9: An abstract view of a CBE-CS implementation framework.

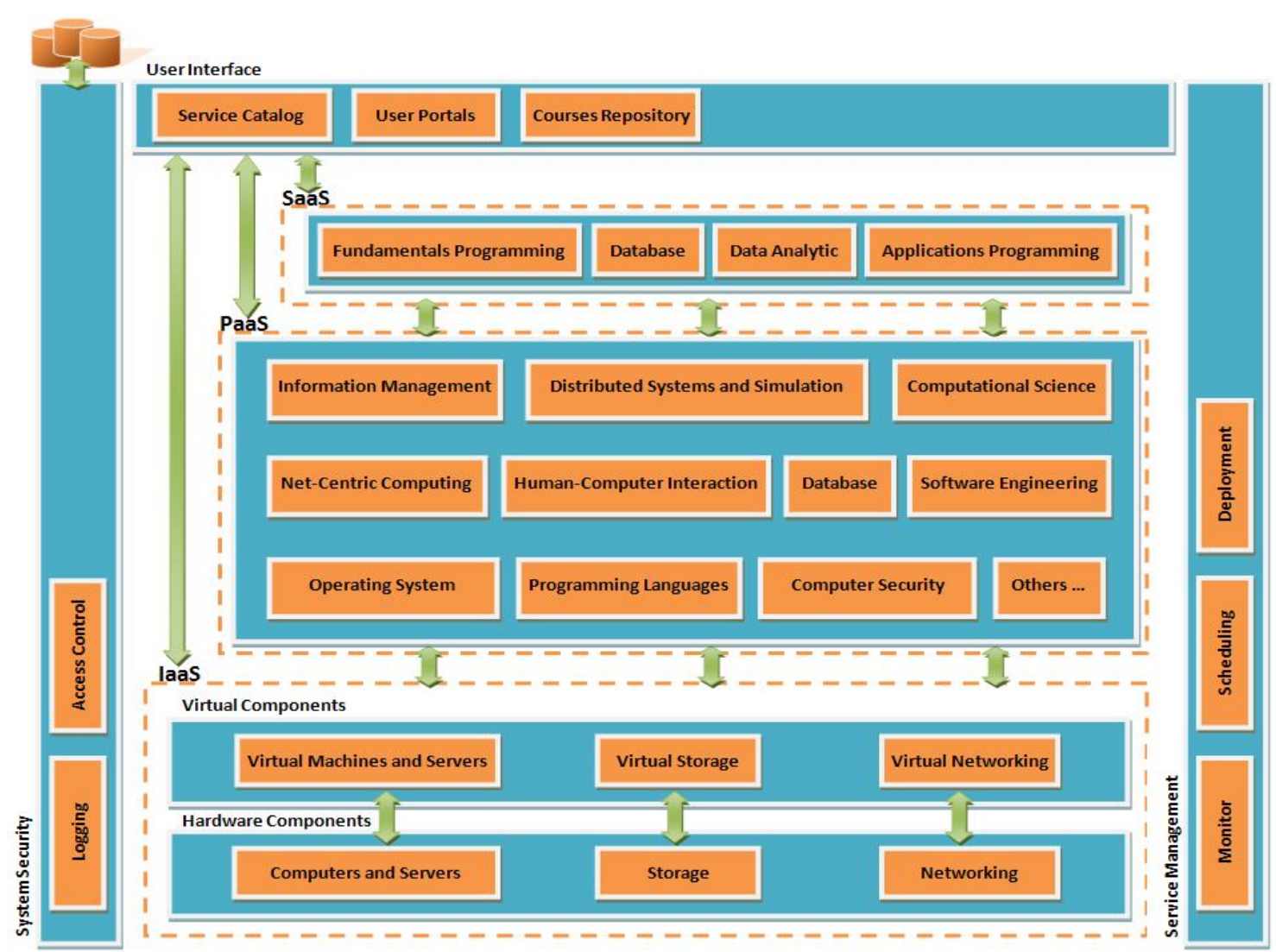

Figure 10: The proposed ecosystem of Cloud-Base Education for Computer Science. 


\section{Concluding Remarks and Future Work}

This paper explored potential benefits of Cloud Computing in educational settings to overcome the limitations of traditional classrooms and e-learning. The work was primarily focused on Computer Science education to improve student learning using the services offered by the Cloud. The paper identified potential CS courses to teach through the Cloud with the ability to add more courses. Further, Cloud-Based Education for Computer Science, CBE-CS, was introduced and the ecosystem of the proposed platform was presented.

This work has highlighted the benefits of the Cloud for institutions, administrators, faculty, and students. Three pilot courses (Database, Operating System, and Parallel Computing) on different public Clouds were discussed and the usefulness of a CBE-CS framework was elaborated. It is argued that Cloud Computing can offer a plethora of tools and options for STEM education.

The proposed framework is a roadmap for implementing a complete virtual classroom ecosystem on the Cloud. An independent study needs to be conducted to compare the learning process using the proposed framework contrast with the traditional learning environment. As part of the future work, the proposed ecosystem needs to be implemented and be evaluated to find out its strengths and limitations. Further, the framework needs to be examined how it can efficiently enhance the learners' performance. Finally, future studies need to investigate the effectiveness of the course contents and their placements in the framework.

\section{Bibliography}

1. Armburst, A. Fox, R. Griffth, A.Joseph, R.Kaltz, G. Lee, D. Patterson, A. Rabkin, and M. Zaharia. "Above the Clouds: A Berkeley View of Cloud Computing". http://www.eecs.berkeley.edu/Pubs/TechRpts/2009/EECS2009-28.pdf

2. Escalante, B. F. (2010). "Cloud Computing Fundamentals". In Handbook of Cloud Computing. Springer link.

3. Delic Walker. "Emergence of the academic computing clouds". ACM publications, August 2008. http://portal.acm.org/citation.cfm?id=1414664

4. M. Chowdhury. "Cloud Computing: Facts, Security, \& Challenges." http://www.aipath.com/mchowdhury_law447b.pdf

5. Dias Marcos, Alexdandre, Buyya. "Evaluating the cost benefits of using the cloud computing to extend the capacity of clusters". ACM Publications 2009. http://portal.acm.org/citation.cfm?id=1551635

6. Gong, C., Liu, J., Zhang, Q., Chen, H. \& Gong, Z. (2010) "The Characteristics of Cloud Computing”, Parallel Processing Workshops (ICPPW), 2010 39th International Conference on 13-16 Sept. 2010, pp. 275 - 279.

7. William Y. Chang, H. A.-A. (2010). "Cloud Service Architecture and Related Standards3,2". In Transforming Enterprise Cloud Services (pp. 87-132). Springer Science+Business Media B.V.

8. NIST, National Institutes of Standards and Technology, http://www.nist.gov/itl/cloud/

9. Terry, D. (2011) “ACM Tech Pack on Cloud Computing”, ACM Tech Pack Committee on Cloud Computing, (October, 2011) http://techpack.acm.org/Cloud/Cloud_computing.pdf

10. Dong, B., Zheng, Q., Yang, J., Li, H. \& Qiao, M. (2009) “An e-Learning Ecosystem Based on Cloud Computing Infrastructure", Ninth IEEE International Conference on Advanced Learning Technologies,

11. Microsoft (2011), Windows Azure Platform, http://www.microsoft.com/azure/default.mspx

12. Amazon (2011) Amazon Elastic Compute Cloud (EC2). http://aws.amazon.com/ec2/

13. Google (2011b), Google Apps, http://code.google.com/appengine/

14. Chen, R. \& Liu, S. (2009) “Applying Concept Mapping on the Influent Learning in Virtual Classroom”, Ninth International Conference on Hybrid Intelligent Systems, 266-270 
15. Das, S., Dhande, A. P. \& Bhoi, M. (2010) “Leveraging Virtual Classroom for Effective Learning”, t4e, pp.101106, 2010 International Conference on Technology for Education, 2010.

16. Wong, D. (2007) “A Critical Literature Review on E-Learning Limitations”, JASA, 2, pp. 55-62

17. Ma, H., Zheng, Z., Ye, F. \& Tong, S. (2010) "The Applied Research of Cloud Computing in the Construction of Collaborative Learning Platform under E-Learning Environment", International Conference on System Science, Engineering Design and Manufacturing Information.

18. Vaquero, L.M. (2011), "EduCloud: PaaS Versus IaaS Cloud Usage for an Advanced Computer Science Course, Education", IEEE Transactions on Education (99) pp.1, January 2011.

19. Al-Zoube, M. (2009) "E-learning on the Cloud", International Arab Journal of e- technology, 1(2). pp.58-64.

20. Vouk, M., Averritt, S., Bugaev, M., Kurth, A., Peeler, A., Shaffer, H., Sills, E., Stein, S. \& Thompson, J. (2008) "Powered by VCL - Using Virtual Computing Laboratory (VCL)", Proc. 2nd International Conference on Virtual Computing (ICVCI), 15-16 May, 2008, RTP, NC, pp 1-10.

21. Buyya, R., Broberg, J. \& Goscinski, A. (2011) “Cloud Computing, Principles and Paradigms", Wiley \& Sons.

22. GoGrid, http://www.gogrid.com/

23. SalesFoce Cloud platform, https://www.salesforce.com/platform/

24. Finke, A. \& Bicans, J. (2010) "E-Learning System Content and Architecture Evolution", 16th International Conference on Information and Software Technologies, Kaunas, Lithuania pp. 311-315.

25. Rajaei, H. (2004) "Distributed Virtual Training Environment", In: Proceedings of 2nd Swedish-American Workshop on Modeling and Simulation (SAWMAS 2004), Feb, FL

26. Brown, A. (2006), "Learning from a Distance", Journal of Property Management, 71(4) pp. 42-45.

27. Stephen, M. \& Gary, J. S. (2006), "Blended Learning, Classroom and E-Learning”, The Business Review, 5(1)

28. Singh, H. (2003), "Building Effective Blended Learning Programs", November - December 2003 Issue of Educational Technology, 43(6) pp. 51-54.

29. Gudanescu, N. (2010), "Using Modern Technology for Improving Learning process at Different Educational Levels", Procedia Social and Behavioral Sciences, 2, pp. 5641-5645

30. Pocatilu, P., Alecu, F. \& Vetrici, M. (2009), "Using Cloud Computing for E-learning Systems", Proceeding Proceedings of the 8th WSEAS international conference on Data networks, communications, computers, ACM, World Scientific and Engineering Academy and Society (WSEAS) Stevens Point, Wisconsin, USA.

31. CS2008 Taskforce, (2008), "Computer Science Curriculum 2008", ACM / IEEE taskforce, www.acm.org/education/curricula/ComputerScience2008.pdf

32. Krook, D. (2011), "Create a PHP development environment on the Cloud, Learn how to use the IBM Cloud to build and test your PHP applications", Copyright IBM Corporation. http://www.ibm.com/developerworks/cloud/library/cl-createphponcloud/

33. Fujimoto, R. M., Malik, A. W. \& Park, A. J. (2010), "Parallel and Distributed Simulation in the Cloud", SCS M\&S Magazine- 3, July, pp. 1-10

34. OpenSSH (2011), http://www.openssh.com,

35. Palmer, R., \& Dodson, L., (2011), "Distance Learning in the Cloud: Using 3G Enabled Mobile Computing to Support Rural Medical Education “, Journal of the Research Center for Educational Technology (RCET), 7(1). 